\title{
Strongly Asymmetric Sequences Generated by Four Elements
}

\author{
By František Fiala*
}

\begin{abstract}
Some general properties of strongly asymmetric sequences generated by $m \geqq 1$ elements $(m-S A S)$ are given. Computational experience with two algorithms-for listing of all 4-SASs of a given length $n$ and for generating the smallest 4-SAS of length $n=1,2, \cdots$ -supports the conjecture that there exists an infinite 4-SAS. The smallest 4-SAS of length 592 is presented.
\end{abstract}

Let us put

(1)

$$
\mathrm{E}_{m}=\{0,1, \cdots, m-1\}
$$

where $m \geqq 1$. A sequence

$$
\left(a_{1}, a_{2}, \cdots, a_{n}\right)
$$

is said to be a strongly asymmetric m-sequence ( $m$-SAS) of length $n$ if

(a) $a_{i} \in \mathrm{E}_{m}$ for $i=1, \cdots, n$;

(b) for any $j \geqq 0, k>0$ such that $j+2 k \leqq n$ two consecutive segments $\left(a_{i+1}, \cdots\right.$, $\left.a_{i+k}\right),\left(a_{i+k+1}, \cdots, a_{i+2 k}\right)$ do not contain the same number (frequency) of 0 's, 1 's, $\cdots$, $(m-1)$ 's.

For instance, if $m=3$ then (0102010) is 3-SAS of length 7, but (01020120) is no 3-SAS (for $j=1, k=3$ two consecutive segments (102), (012) contain the same number of 0 's, 1's and 2's).

An infinite sequence

$$
\left(a_{1}, a_{2}, \cdots, a_{n}, \cdots\right)
$$

is $m$-SAS if for an arbitrary positive integer $n$ the sequence (2) is $m$-SAS.

P. Erdös [1] posed the problem of finding an infinite SAS using the minimal number of symbols. A. A. Evdokimov [2] constructed an infinite $m$-SAS for $m=25$ and expressed the opinion that the number $m$ might be reduced. It is easy to establish that there is no infinite $m$-SAS for $m \leqq 3$ [2]. Therefore, I investigated the case $m=4$. It appears that the technique used in [2] is not applicable to this case. In this paper some general properties of $m$-SASs are given and computational experience with 4-SASs is collected. The smallest 4-SAS of length 592 is presented.

1. We shall describe some general properties of $m$-SAS for $m \geqq 1$ which can be easily verified.

Received June 22, 1970.

AMS 1969 subject classifications. Primary 1007, 0510; Secondary 6510.

Key words and phrases. Strongly asymmetric sequences, lexicographical ordering, number systems.

* This paper was written while the author was a post-doctorate fellow at the Department of Computing Science, University of Alberta, Edmonton, Alberta, Canada.

Copyright @ 1971, American Mathematical Society 
1.1. An $m$-SAS

$$
\left(a_{1}, \cdots, a_{n_{1}}\right)
$$

has a prolongation if there is an $m$-SAS

$$
\left(a_{1}, \cdots, a_{n_{1}}, a_{n_{1}+1}, \cdots, a_{n_{2}}\right)
$$

of length $n_{2}>n_{1} . m$-SAS (4) is called an initial segment of (5).

The set of all (finite and infinite) $m$-SASs can be ordered lexicographically, i.e. if

$$
\begin{aligned}
& \left(a_{1}^{\prime}, \cdots, a_{n_{1}}^{\prime}\right), \\
& \left(a_{1}^{\prime \prime}, \cdots, a_{n_{3}}^{\prime \prime}\right),
\end{aligned}
$$

are two $m$-SASs then (6) is less than (7) if either there exists such an $i, 0 \leqq i<$ $\min \left(n_{1}, n_{2}\right)$, that

$$
a_{i}^{\prime \prime}=a_{i}^{\prime \prime} \quad \text { for } \quad j=1, \cdots, i
$$

and

$$
a_{i+1}^{\prime}<a_{i+1}^{\prime \prime}
$$

or (6) is an initial segment of (7). E.g. (01020) is less than (0121030), (10230) is less than (120).

Principle of duality. If (2) is an $m$-SAS then

$$
\left(m-1-a_{1}, \cdots, m-1-a_{n}\right)
$$

is $m$-SAS, too. (10) is called dual to (2) and vice versa.

All $m$-SASs of a given length $n$ form a finite chain in the lexicographical ordering. If (2) is the smallest $m$-SAS of length $n$ then its dual $m$-SAS is the greatest one (of the same length).

Obviously, there is an m-SAS of length $n$ if and only if there is the smallest $m$-SAS of length $n$.

1.2. If (2) is an $m$-SAS of length $n$ and $p$ is an arbitrary permutation of $\mathbf{E}_{m}$ then

$$
\left(p\left(a_{1}\right), p\left(a_{2}\right), \cdots, p\left(a_{n}\right)\right)
$$

is $m$-SAS, too. If (2) contains each element of $\mathbf{E}_{m}$ and $p$ is nonidentical then

$$
\left(p\left(a_{1}\right), \cdots, p\left(a_{n}\right)\right) \neq\left(a_{1}, \cdots, a_{n}\right) .
$$

1.3. Let $m \leqq 3$. Direct check shows that there is no $m$-SAS of length $n>7$. Therefore, any 4-SAS of length $n \geqq 8$ contains all numbers $0,1,2,3$. Consequently, the number $S_{n}^{4}$ of all 4-SASs of length $n \geqq 8$ is divisible by $4 !=24$. For arbitrary $m>1$, the number $S_{n}^{m}$ of all $m$-SASs of length $n \geqq 2$ is divisible by $m(m-1)$.

1.4. Denote by

$$
\left(a_{1}, \cdots, a_{2^{m-1}}\right)
$$

the smallest $m$-SAS of length $2^{m}-1$. Then (13) has no $m$-SAS prolongation and

$$
\left(a_{1}, \cdots, a_{2^{m-1}}, m, a_{1}, \cdots, a_{2^{m-1}}\right)
$$

is the smallest $(m+1)$-SAS (of length $\left.2^{m+1}-1\right)$. 
Thus, 0, 010, 0102010, 010201030102010, 0102010301020104010201030102010 are the smallest 1-, 2-, 3-, 4- and 5-SASs, respectively.

Proof by induction. For $m=1$ the proposition obviously holds. Assume that it is true for $m \geqq 1$, i.e. (13) has no $m$-SAS prolongation and (14) is the smallest $(m+1)$-SAS. We prove that the proposition holds for $m+1$.

Let $\left(a_{1}, \cdots, a_{2^{m-1}}, m, a_{1}, \cdots, a_{2^{m-1}}, a\right)$ be an ( $\left.m+1\right)$-SAS prolongation of (14). If $a<m$ then $\left(a_{1}, \cdots, a_{2^{m}-1}, a\right)$ is an $m$-SAS prolongation of (13), contrary to our assumption. $a=m$ is obviously impossible. Thus (14) has no $(m+1)$-SAS prolongation.

It is easy to check that

(15) $\left(a_{1}, \cdots, a_{2^{m-1}}, m, a_{1}, \cdots, a_{2^{m-1}}, m+1, a_{1}, \cdots, a_{2^{m-1}}, m, a_{1}, \cdots, a_{2^{m-1}}\right)$ is an $(m+2)$-SAS of length $2^{m+2}-1$. Let

$$
\left(b_{1}, \cdots, b_{2^{m+2-1}}\right)
$$

be the smallest $(m+2)$ )-SAS (of length $\left.2^{m+2}-1\right)$. Then $\left(b_{1}, \cdots, b_{2^{m+1-1}}\right)$ is an $(m+2)$-SAS and therefore

$$
\left(b_{1}, \cdots, b_{2^{m+1-1}}\right) \leqq\left(a_{1}, \cdots, a_{2^{m-1}}, m, a_{1}, \cdots, a_{2^{m-1}}\right) .
$$

(14) is the smallest $(m+1)$-SAS and also the smallest $(m+2)$-SAS. Hence, in the relation (17), the equal sign holds. (14) has no $(m+1)$-SAS prolongation, thus $b_{2^{m+1}}=m+1$. Again

$$
\left(b_{2^{m+1}+1}, \cdots, b_{2^{m+2-1}}\right) \geqq\left(a_{1}, \cdots, a_{2^{m-1}}, m, a_{1}, \cdots, a_{2^{m-1}}\right) .
$$

The minimal property of (16) causes again that in (18) the equal sign holds. Thus $(15)$ is the smallest $(m+2)$-SAS.

2. We consider the case $m=4$. Two algorithms have been prepared. The first one lists all 4-SASs of a given length $n$ in their lexicographical order. The second algorithm generates the smallest 4-SAS of length $n=1,2, \cdots$. Both of them were written in FORTRAN IV for IBM 360/67.

2.1. We recall that the set of all 4-SASs of a given length $n$ is ordered lexicographically (1.1). A 4-SAS of length $n$

$$
\left(a_{1}, a_{2}, \cdots, a_{n}\right)
$$

may be seen as an ( $n$-positional) integer written in the number system with the radix 4. Therefore, we can also handle (2) as a number and add to it another number (in the number system with the radix 4). This feature is used in the following algorithms both for finding all 4-SASs of length $n$ in their ascendent order and for generating the smallest 4-SAS (2.2).

The first algorithm runs as follows:

2.1.1. Start with the smallest 4-SAS of the form (2).

2.1.2. Write the current 4-SAS.

2.1.3. Add 1 to the current sequence (in the number system with the radix 4). If a transfer from the most left position occurs, go to 2.1.5.

2.1.4. Ask if the new sequence is 4-SAS. If the answer is "Yes", go to 2.1.2. If the answer if "No", go to 2.1.3.

2.1.5. Stop. 
A complete listing of 4-SASs was made for $n=1, \cdots, 9$. The numbers $S_{n}^{4}$ of all 4-SASs for $n=10, \cdots, 18$ were found using 1.3.

The following tables show some of the results. Table 1 contains the first 25 smallest 4-SASs of lengths $14,15,16$. Table 2 shows the numbers $S_{n}^{4}$ and the ratio of two consecutive $S_{n}^{4}, S_{n+1}^{4}$. For comparison, similar data for $m=3$ are added.

For $n<14$, every 4-SAS has at least one prolongation, but for $n \geqq 14$, there are some 4-SAS without any prolongation (denoted by * in Table 1).

2.2. The algorithm for generating the smallest 4-SAS for $n=1,2, \cdots$ can be described in the following way (cf. 2.1):

2.2.1. Start with $n=1,\left(a_{1}\right)=(0)$.

2.2.2. Write $n,\left(a_{1}, \cdots, a_{n}\right)$.

2.2.3. Multiply the number $a_{1} \cdots a_{n}$ by 4 (in the number system with the radix 4). Add 1 to $n$.

2.2.4. Ask if the new sequence is 4-SAS. Yes: go to 2.2.2. No: go to 2.2.5.

TABLE 1

\begin{tabular}{|c|c|c|c|}
\hline $\begin{array}{c}\text { Order } \\
\text { No. }\end{array}$ & $n=14$ & $n=15$ & $n=16$ \\
\hline 1 & 01020103010201 & $010201030102010 *$ & 0102010301021013 \\
\hline 2 & 01020103010210 & 010201030102101 & 0102010301021230 \\
\hline 3 & 01020103010212 & 010201030102123 & 0102010301021231 \\
\hline 4 & 01020103010213 & 010201030102131 & 0102010301021232 \\
\hline 5 & $01020103012010 *$ & 010201030102132 & 0102010301021310 \\
\hline 6 & 01020103012013 & 010201030120131 & 0102010301021312 \\
\hline 7 & 01020103012023 & 010201030120230 & 0102010301021320 \\
\hline 8 & 01020103012101 & 010201030120232 & 0102010301021321 \\
\hline 9 & 01020103012103 & 010201030121012 & 0102010301021323 \\
\hline 10 & 01020103012130 & 010201030121013 & 0102010301201310 \\
\hline 11 & 01020103012131 & 010201030121031 & 0102010301201312 \\
\hline 12 & 01020103012132 & 010201030121301 & 0102010301202302 \\
\hline 13 & 01020103012310 & 010201030121303 & 0102010301202303 \\
\hline 14 & 01020103012312 & 010201030121310 & 0102010301202320 \\
\hline 15 & 01020103012313 & 010201030121312 & 0102010301202321 \\
\hline 16 & 01020103012320 & 010201030121321 & 0102010301210123 \\
\hline 17 & 01020103012321 & 010201030121323 & 0102010301210130 \\
\hline 18 & 01020103020102 & 010201030123101 & 0102010301210131 \\
\hline 19 & 01020103020120 & 010201030123103 & 0102010301210132 \\
\hline 20 & 01020103020121 & 010201030123121 & 0102010301210310 \\
\hline 21 & 01020103020123 & 010201030123130 & 0102010301210312 \\
\hline 22 & 01020103021013 & 010201030123202 & 0102010301210313 \\
\hline 23 & $01020103021020^{*}$ & 010201030123203 & $0102010301213010^{*}$ \\
\hline 24 & 01020103021023 & 010201030123212 & 0102010301213012 \\
\hline 25 & 01020103021202 & $010201030201020^{*}$ & 0102010301213013 \\
\hline
\end{tabular}


2.2.5. Add 1 to the current sequence (in the number system with the radix 4). If a transfer from the most left position occurs, go to 2.2.6. Elsewhere, go to 2.2.4.

2.2.6. Stop.

In other words, if $\left(a_{1}, \cdots, a_{n}\right)$ is the smallest 4-SAS of length $n$, we will try to prolong it successively by adjoining to the end $0,1,2,3$. The first prolongation

TABLE 2

\begin{tabular}{|c|c|c|c|c|}
\hline Length & $S_{n}^{4}$ & $S_{n+1}^{4} / S_{n}^{4}$ & $S_{n}^{3}$ & $S_{n+1}^{3} / S_{n}^{3}$ \\
\hline 1 & 4 & & 3 & \\
\hline 2 & 12 & 3 & 6 & \\
\hline & & 3 & & 2 \\
\hline 3 & 36 & $2 . \overline{6}$ & 12 & 1.5 \\
\hline 4 & 96 & 97 & 18 & $\overline{-}$ \\
\hline 5 & 264 & $\begin{array}{r}2.75 \\
-\end{array}$ & 30 & 1.0 \\
\hline 6 & 648 & $\begin{array}{c}2.45 \\
-\end{array}$ & 30 & \\
\hline & & 2.4 & 18 & 0.6 \\
\hline 7 & 1584 & $2.2 \overline{57}$ & 18 & \\
\hline 8 & 3576 & 2.201342282 & None & \\
\hline 9 & 7872 & & & \\
\hline 10 & 15360 & 19 & & \\
\hline 11 & 29184 & & & \\
\hline 12 & 51120 & 1.768075117 & & \\
\hline 13 & 90384 & & & \\
\hline 14 & 158448 & 1.806876704 & & \\
\hline 15 & 286296 & & & \\
\hline 16 & 509808 & 1.773797194 & & \\
\hline 17 & 904296 & 1.721011704 & & \\
\hline 18 & 1556304 & & & \\
\hline
\end{tabular}


TABLE 3 01020103010210131012132021013010203020120231012023
20212303230102030201213010203013032131012101301020
10302303101201032021202320130201312130313212023020
13230313032010203021013121020301323013121012132010
20302102321201303230131210121320132302010231232023
21201213010201030212302101310121321202123130102010
30213230213013120312320231210121310232131013121030
10231323023101312320210130102101303202123032301020
13121303132021231303132302321201032313201213121013
01021230123130312320231210203013123130323031012320
30102010310121310302012312102032021020313032021303
132101213120103010203213230121013021231210

(if any) is the smallest 4-SAS of the length $n+1$. If there is no prolongation of $\left(a_{1}, \cdots, a_{n}\right)$ then we take instead of it the following greater 4-SAS of length $n$ (with regard to the lexicographical order-cf. 2.1) and proceed in a similar way. The procedure stops if no 4-SAS of length $n$ has any prolongation.

Some of the results obtained by using this algorithm follow.

The longest 4-SAS I found is the following smallest 4-SAS of length 592:

The preceding 4-SAS was not found at once and Table 4 shows the necessary computing time for generating the smallest 4-SASs for different lengths $n$ :

We can see that for increasing $n$, the indicated computing time increases very rapidly. This increase is partially due to the increase in length, but mostly it is caused by the fact that we do not have available for a given $n$ all lexicographically ordered 4-SASs of length $n$. If a 4-SAS has no prolongation then we have to determine the next greater 4-SAS of the same length and try to prolong that one. This procedure is very costly with regard to time. Table 5 lists the lengths $n<592$ having the prop-

TABLE 4

\begin{tabular}{l|r|r|r|r|r|r|r|r}
\hline Length $n$ & 50 & 100 & 200 & 261 & 299 & 376 & 434 & 466 \\
\hline Time (min) & 0.17 & 0.60 & 2.57 & 5.57 & 10.57 & 25.57 & 40.57 & 55.57 \\
\hline
\end{tabular}

\begin{tabular}{l|c|c|c|c}
\hline Length $n$ & 509 & 530 & 588 & 592 \\
\hline Time (min) & 70.57 & 85.57 & 100.57 & 115.57 \\
\hline
\end{tabular}


TABLE 5

15(1), 23(1), 36(1), 62(2), 63(3), 85(1), 94(1),

106(1), 119(2), 122(2), 124(1), 130(1), 135(1), 136(1), 140(1), 146(2), 149(1), 153(1), 154(1), 167(1), 172(1), 176(1), 177(1), 187(4), 188(1), $189(1)$,

205(1), 216(3), 222(1), 224(1), 239(1), 240(1), 241(1), 243(1), 248(1), 249(1), 282(1), 283(4), 284(2), 289(2), 290(1), 299(3),

305(1), 306(1), 308(1), 310(2), 314(1), 321(1), 323(3), 326(1), 331(6), 332(1), 335(1), 339(1), 343(1), 358(1), 363(1), 369(2), 370(4), 373(1), 375(1), 376(1), 390(1), 392(2), 394(2), 395(3),

404(1), 412(1), 415(1), 420(1), 424(1), 428(2), 434(1), 435(1), 445(2), 446(1), 447(2), 449(1), 456(1), 462(2), 463(1), 464(1), 470(3), 473(2), 478(2), 484(1), 485(1), 490(1), 491(2),

508(1), 509(1), 510(2), 515(1), 525(1), 529(1), 530(1), 532(3), 542(1), 543(1), 544(1), 549(1), 554(2), 559(1), 574(1), 583(1), 588(1), 589(1), 591(1), 592(?)

erty that the smallest 4-SAS of length $n$ has no prolongation. The number $i$ in parentheses shows that $i$ smallest 4-SASs of length $n$ (with regard to the lexicographical order) have no prolongation.

The most unfavorable case occurs for $n=331$ when 6 smallest 4-SASs have no prolongation. But the most frequent case is that only one smallest 4-SAS has no prolongation.

Frequency of symbols in the smallest 4-SAS of length 592 and several consecutive segments is given in Table 6 . We can see from the second column that the frequency of an arbitrary symbol from $E_{4}$ in a 4-SAS (or its segment) of length 100 varies between 17 and 35 .

TABLE 6

\begin{tabular}{c|c|c|c|c|c|c|c}
\hline & \multicolumn{7}{c}{ Length } \\
\cline { 2 - 7 } Symbol & $1-100$ & $101-200$ & $201-300$ & $301-400$ & $401-500$ & $501-592$ & $1-592$ \\
\hline 0 & 35 & 29 & 26 & 24 & 20 & 26 & 160 \\
1 & 26 & 25 & 27 & 27 & 27 & 26 & 158 \\
2 & 22 & 23 & 28 & 24 & 24 & 21 & 142 \\
3 & 17 & 23 & 19 & 25 & 29 & 19 & 132 \\
\hline
\end{tabular}


Conclusions. Unfortunately, the problem of determining an infinite 4-SAS remains open. This paper is only a small contribution toward its solution. From Table 2 we can see that the number of 4-SASs of length $n$ grows quickly for small $\boldsymbol{n}$ and will probably continue. In spite of the fact that the time for generating the smallest 4-SAS increases for greater $n$ (Table 4) the prolongation is not more difficult in essence (Tables 5, 6). That leads to the following conjecture:

The algorithm 2.2 will never stop, i.e. for any $n=1,2, \cdots$ there exists a 4-SAS.

Acknowledgements. I am very indebted to Dr. I. Rosenberg for introducing me to this problem and for many suggestions and discussions about it.

Department of Computing Science

University of Alberta

Edmonton, Alberta, Canada

1. Erdös, "Some unsolved problems," Magyar Tud. Akad. Mat. Kutató Int. Közl., v. 6, 1961, pp. 221-254; Russian transl., Matematika, v. 7, 1963, no. 4, p. 128. MR 31 \#2106.

2. A. A. EvDokimov, "Strongly asymmetric sequences generated by a finite number of symbols," Dokl. Akad. Nauk SSSR, v. 179, 1968, pp. 1268-1271 = Soviet Math. Dokl., v. 9, 1968, pp. 536-539. MR 38 \#3156. 